\title{
Dextran sulfate sodium administered orally is depolymerized in the stomach and induces cell cycle arrest plus apoptosis in the colon in early mouse colitis
}

\author{
YOSHIO ARAKI $^{1}$, TADAO BAMBA ${ }^{2}$, KEN-ICHI MUKAISHO $^{1}$, OSAMU KANAUCHI $^{4}$, \\ HIROMITSU BAN ${ }^{2}$, SHIGEKI BAMBA ${ }^{2}$, AKIRA ANDOH ${ }^{3}$, YOSHIHIDE FUJIYAMA ${ }^{2}$, \\ TAKANORI HATTORI $^{1}$ and HIROYUKI SUGIHARA ${ }^{1}$
}

\author{
Departments of ${ }^{1}$ Pathology, ${ }^{2}$ Internal Medicine and ${ }^{3}$ Division of Mucosal Immunology, Shiga University of \\ Medical Science, Seta Tsukinowa, Otsu, Shiga 520-2192; ${ }^{4}$ Central Laboratories for Frontier Technology, \\ Kirin Holdings Co., Ltd., 1-13-5, Kanazawa-ku, Yokohama, Kanagawa 236-0004, Japan
}

Received April 9, 2012; Accepted July 6, 2012

DOI: 10.3892/or.2012.1969

\begin{abstract}
The mechanisms responsible for human inflammatory bowel disease remain poorly understood. The pathogenic factors for dextran sulfate sodium (DSS)-induced colitis, one of the experimental animal colitis models, also remain unknown. Furthermore, detailed studies on DSS metabolism in the gut lumen have not been reported. Therefore, we investigated DSS metabolism in the mouse gut lumen and report the mechanisms which induce colitis. DSS was labeled with 2-aminopyridine (pyridylamino-DSS, PA-DSS). PA-DSS was administered orally to male BALB/cA Jcl mice. The metabolites and histological findings were observed using HPLC and light or fluorescence microscopy. PA-DSS with Mr 5000 was depolymerized rapidly in the gastric lumen, and the depolymerized PA-DSS was absorbed in the small intestine. Therefore, the majority of the PA-DSS in the cecal contents returned to $\mathrm{Mr}_{\mathrm{r}} 5000 \mathrm{PA}-\mathrm{DSS}$, escaping absorption in the small intestine. $\mathrm{Mr}_{\mathrm{r}} 5000$ DSS induced severe colitis, and immunostaining using an anti-mouse Ki-67 antibody and the TUNEL assay showed that DSS arrested the cell cycle at the $\mathrm{G}_{0}$ phase and induced apoptosis of the colonic epithelium. Mr 2500 PA-DSS, however, induced these same effects weakly. During these processes, we observed that the epithelial cells can depolymerize DSS themselves. An in vitro study using Caco-2 cells also showed similar effects. Mr 5000 DSS was depolymerized in the gut lumen and epithelial cells. Therefore, the molecular
\end{abstract}

Correspondence to: Dr Yoshio Araki, Department of Pathology, Shiga University of Medical Science, Seta Tsukinowa, Otsu, Shiga 520-2192, Japan

E-mail: yir0828@belle.shiga-med.ac.jp

Key words: apoptosis, cell cycle arrest, crypt disappearance, depolymerization, dextran sulfate sodium, DSS-induced colitis, molecular mass mass distribution of the DSS differed between each part in the lumen. As an early stage event, DSS induced colitis through cell cycle arrest and apoptosis according to its molecular mass.

\section{Introduction}

In human inflammatory bowel disease (IBD), the subtypes of ulcerative colitis (UC) and Crohn's disease are chronic, relapsing, and remitting conditions that characterized by diarrhea, bloody stools, abdominal pain and weight loss. Currently, UC is believed to be caused by multiple factors, including genetic and environmental factors. Histologically, UC is characterized by crypt abscesses, crypt distortion and loss, ulceration, and by the infiltration of large numbers of neutrophils, monocytes and lymphocytes. IBD affects at least 1 in 1,000 people in Western countries (1). Most importantly, UC is associated with an increased risk of colorectal cancer (2). Despite many investigations into IBD, its etiology and pathogenic mechanisms remain poorly understood.

Experimental animal models of colitis have been developed in order to investigate the underlying physiologic mechanisms and to improve medical therapies for IBD. In the most commonly-used models, colitis is induced by administering sulfated polysaccharides such as dextran sulfate sodium (DSS) or carrageenan (3). In general, the history of the development of DSS-induced colitis has paralleled the history of antipepsin agents. Since the mucosal protection theory for gastric mucus was suggested, many antipepsin agents have been developed. More recently, amylopectin sulfate was developed in the United States, and DSS was developed in Sweden. At the same time, the major side effects of antipepsin agents were discovered, and they were found to induce intestinal and cecal ulceration in animal experiments (4). Hence, DSS has been used for the production of experimental colitis. DSS is a heparin-like polysaccharide consisting of anhydro-D-glucose ( $\alpha-1,6$-glucosidic linkage) moieties and sulfate. DSS contains approximately $17 \%$ sulfur, with up to three sulfate groups per glucose molecule (5) (Fig. 1A). DSS induced-colitis exhibits some of the clinical 
and histological features of UC. For example, DSS-induced colitis starts from the rectum and subsequently spreads to the anal side. Histologically, DSS-induced colitis is characterized by crypt distortion and loss, ulceration, and the infiltration of large numbers of inflammatory cells, which is similar to UC. In addition, the dysplasia and adenocarcinomas observed in UC patients are also recognized in this mode $(6,7)$. It has been reported that the development of colitis is dependent on the molecular mass and sulfation of the DSS, in addition to the dosage and duration of the administration $(8,9)$. However, it is unknown how DSS actually induces colitis. Therefore, an investigation into the pathogenic factors in this colitis model may help elucidate the mechanisms responsible for human UC. Despite many studies, the pathogenic factors in DSS-induced colitis remain unknown, although DSS has been often used as a UC model. Even with respect to the metabolism of DSS in the gut lumen, there have been no detailed studies. Therefore, the main purpose of this study was to investigate the metabolism of DSS in the gut lumen. In the present study, we used 2-aminopyridine labeling and HPLC for detecting DSS in biological materials according to our previous reports $(10,11)$. Additionally, we also propose a plausible mechanism for DSS-induced colitis based on our findings for the unique metabolism of DSS.

\section{Materials and methods}

Chemicals. Mr 5000 and 500k DSS and 2-aminopyridine were obtained from Wako Pure Chemical Industries, Ltd. (Osaka, Japan). Mr 8000, 10000 DSS and D-glucose 3-sulfate were obtained from Sigma Chemical Co. (St. Louis, MO). Mr 2500 DSS was obtained from Tokyo Chemical Industry Co., Ltd. (Tokyo, Japan).

Labeling of DSS. The pyridylamination of the reducing termini of sugar chains has been useful for the structural analysis and metabolic studies on $\mathrm{N}$ - or $\mathrm{O}$-glycosidically-linked sugar chains. The labeling of each molecular mass of DSS using 2-aminopyridine was carried out according to our previous report $(10,11)$. We obtained pure pyridylamino-DSS (PA-DSS) of each molecular mass, and the PA-D-glucose 3-sulfate as a PA-monomer.

HPLC conditions. Since PA-DSS is strongly negatively charged in aqueous solution, a strong interaction between PA-DSS and the stationary phase is present. Therefore, $0.2 \mathrm{M}$ phosphate buffer at $\mathrm{pH} 3.0$ was used as the mobile phase according to our previous report $(10,11)$. The mobile phases were delivered isocratically at a flow rate of $1.0 \mathrm{ml} / \mathrm{min}$. We used an HPLC LC6A apparatus (Shimadzu, Kyoto, Japan). The retention time of DSS was determined by gel filtration chromatography on a Cosmosil 5Diol-120 Packed column $\left(7.5 \times 300 \mathrm{~mm}\right.$; Nacalai Tesque Inc., Kyoto, Japan) at $60^{\circ} \mathrm{C}$. For the detection, the fluorescence detector RF-535 (Shimadzu) was used at excitation and emission wavelengths of $320 \mathrm{~nm}$ and $400 \mathrm{~nm}$, respectively.

Animals. Specific pathogen-free male BALB/cA Jcl mice, 6-week old, were purchased from Nippon Clea Inc. (Tokyo, Japan). They were housed in a room with controlled tempera- ture $\left(20-22^{\circ} \mathrm{C}\right)$, humidity $(50-60 \%)$ and a preset light-dark cycle (12 h:12 h). This study was carried out in strict accordance with the recommendations in the Guide for the Care and Use of Laboratory Animals of the National Institutes of Health. The protocol was approved by the Animal Care and Use Committee of the Shiga University of Medical Science (Permit no: 2006-7-6). All surgery was performed under sodium pentobarbital anesthesia, and all efforts were made to minimize suffering.

Mouse PA-DSS-induced colitis. At the beginning of the experiment, the mice were fed the standard diet (MF, Oriental Yeast Co,. Ltd., Tokyo, Japan) containing 5\% (w/w of diet) Mr 5000 or 2500 PA-DSS for 8 days $(n=5)$. During the experimental period, body weight was measured every other day. On the final day, blood samples were collected by cardiac puncture. The contents of the gut lumen were removed, diluted adequately by PBS, centrifuged to remove any insoluble material (14,000 rpm, for $20 \mathrm{~min})$, and then the supernatants were used for the HPLC analysis. Each organ was resected, irrigated with chilled PBS, placed in $0.5 \mathrm{ml}$ of a hexadecyltrimethylammonium bromide solution $(0.5 \%, \mathrm{w} / \mathrm{w})$, homogenized, sonicated and subjected to three rapid cycles of freezing and thawing. The samples were then centrifuged and the supernatants were used for HPLC analysis. The blood samples were centrifuged, and the supernatants were used for HPLC analysis. On the other hand, a specimen at $2 \mathrm{~cm}$ distance from the anal margin (middle colon) was removed, frozen and then cut into $5 \mu \mathrm{m}$ sections. The sections were observed under a fluorescence microscope, or stained with hematoxylin and eosin (H\&E) to observe under light microscopy. The mucosal damage was determined according to a previously described method (12). Briefly, the following three parameters were used: surface epithelium loss, crypt destruction and inflammatory cell infiltration into the mucosa. A score of 0-4 was assigned to each of three parameters according to the extent and the severity of the changes. The sum of the scores from the three parameters represented the mucosal damage score in each animal.

Caco-2 cell culture. Caco-2 (a human colon cancer cell line) cells were purchased from the American Type Culture Collection (Rockville, USA). The cells were cultured in Dulbecco's modified Eagle's minimum essential medium (DMEM, pH 7.4) supplemented with $25 \mathrm{mM}$ glucose, $10 \%$ inactivated fetal bovine serum (FBS), $1 \%$ penicillin-streptomycin and $1 \%$ non-essential amino acid solution at $37^{\circ} \mathrm{C}$ in a humidified $5 \% \mathrm{CO}_{2}$ atmosphere.

Findings of Caco-2 cells using microscopy. We reported that the $\mathrm{Mr}_{\mathrm{r}} 5000$ DSS was depolymerized to $\mathrm{Mr}_{\mathrm{r}} 1800$, and 70\% of the sulfate groups were depleted from each DSS molecule following autoclave treatment (at $115^{\circ} \mathrm{C}$ for $15 \mathrm{~min}, 1.7 \mathrm{~atm}$ ) (13). Therefore, filter sterilization is recommended. The Caco-2 cells were incubated on culture slides. After the cells reached confluence, they were incubated with $3 \% \mathrm{Mr} 5000$ PA-DSS in a growth medium for $24 \mathrm{~h}$. Next, the cells were washed with PBS and incubated with growth medium alone for $24 \mathrm{~h}$. Then, the cells were fixed with ethanol, permeated with acetone, stained with a $0.05 \%$ toluidine blue solution for 

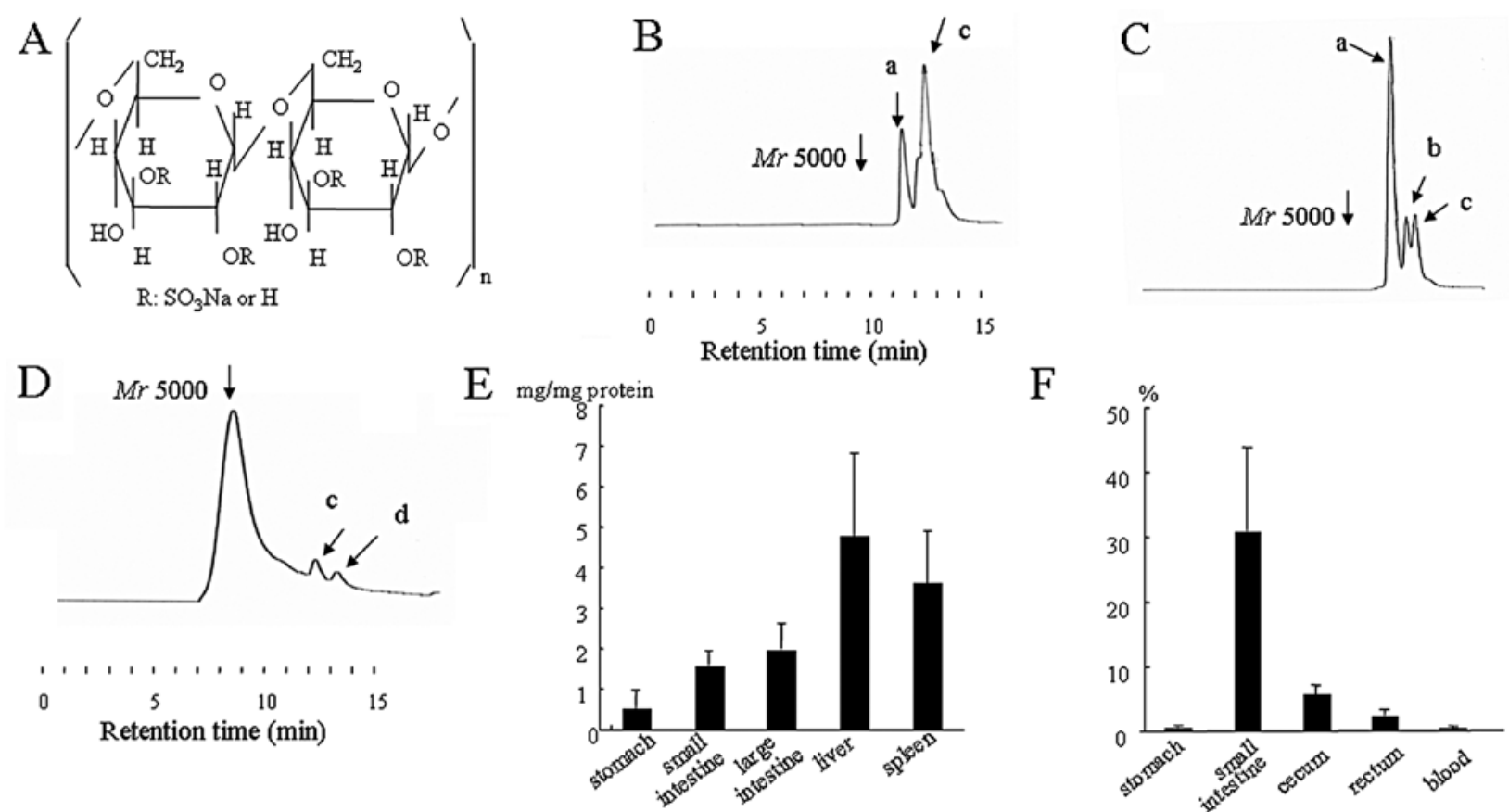

Figure 1. Metabolism of $\mathrm{M}_{\mathrm{r}} 5000$ PA-DSS. (A) Structure of DSS. DSS is a sulfated polysaccharide consisting of anhydro-D-glucose ( $\alpha$-1,6-glucosidic link) and sulfate. (B) Chromatogram of PA-DSS in the gastric contents. The molecular masses were approximately $\mathrm{M}_{\mathrm{r}} 2000$ (peak a) and 1200 (peak c), respectively. (C) Chromatogram of PA-DSS in the contents from the small intestine. The molecular masses were approximately Mr 2000 (peak a), 1500 (peak b), and 1200 (peak c), respectively. (D) Chromatogram of PA-DSS in the cecal contents. A main peak was recognized at approximately Mr 5000 . Smaller peaks of Mr 1200 (peak c) and 750 PA-DSS (peak d) were also recognized. (E and F) Quantification of PA-DSS in each organ and content, respectively. These data show the amount of $\mathrm{M}_{\mathrm{r}} 5000$ PA-DSS regardless of the degree of depolymerization. All values are expressed as means \pm SEM.

$20 \mathrm{~min}$ and observed under light microscopy. The cells were also washed, fixed in $1 \%$ glutaraldehyde and observed under a fluorescence microscope (Labophot-2, Nikon, Tokyo, Japan).

Metabolism of PA-DSS in Caco-2 cells. When confluent and allowed to mature on permeable inserts, the Caco-2 monolayer formed tight junctions and attained many of the morphological and functional characteristics of the intestinal epithelium with normal barrier function (14). We investigated the metabolism of Mr 5000 PA-DSS during passage through this monolayer. Briefly, the Caco-2 cells were plated on Millicell-HA $0.4 \mu \mathrm{m}$ permeable filters (12-mm) (Millipore, Bedford, MA). The transepithelial electrical resistance (TEER) of these cells was measured with an electrical resistance system, Millicell-ERS (Millipore), according to our previous methods (15). Cells with stable TEER readings $>500 \Omega \mathrm{cm}^{2}$ were used (4-5 weeks post plating). A $250 \mu \mathrm{l}$ aliquot of $1 \% \mathrm{Mr} 5000$ PA-DSS solution was inserted inside the porous filters, and $500 \mu \mathrm{l}$ of medium outside of the porous filters was collected at the indicated times and analyzed by HPLC.

Cell cycle arrest and apoptosis in DSS-induced colitis and Caco-2 cells. We observed each sample using immunohistochemical staining. Anti-mouse or anti-human Ki-67 antibodies (Dako Cytomation, Denmark) and the TdT-FragEL ${ }^{\text {TM }}$ DNA Fragmentation Detection kit (Calbiochem, USA) were used to analyze the cell cycle and apoptosis, respectively according to the manuals, provided by the manufacturers.

Statistical analysis. The results are presented as means \pm SEM. The variance was analyzed by the F test. Subsequently, the
Student's t-test for unpaired values was performed to compare the means of the normally distributed data. The Mann-Whitney $\mathrm{U}$ test was also performed to compare the means of nonparametric or abnormally distributed data. Differences were regarded as statistically significant if the P-values were $<0.05$.

\section{Results}

Metabolism of Mr 5000 PA-DSS. First, we investigated the metabolism of DSS in the gut lumen using 2-aminopyridine labeling and HPLC systems. No PA-DSS was recognized in the esophageal mucosa. Surprisingly, Mr 5000 PA-DSS was depolymerized in the gastric contents (Fig. 1B). The two main peaks were speculated to be $\sim \operatorname{Mr} 2000$ (peak a) and 1200 (peak c), according to the relationship between the retention time and the molecular masses of Mr 5000, 10000 PA-DSS and PA-D-glucose-3-sulfate, respectively. The molecular mass distribution in the gastric mucosa was the same as in the gastric contents. Both in the contents and mucosa of the small intestine, the three main peaks were speculated to be approximately Mr 2000 (peak a), 1500 (peak b) and 1200 (peak c), respectively (Fig. 1C). However, the main peaks in the contents of the cecum were speculated to be $\mathrm{Mr} 5000$ (Fig. 1D). Small peaks of Mr 1200 (peak c) and 750 (peak d) were also recognized. In the cecal mucosa, the main peaks were approximately $\mathrm{Mr}$ 2000, 1500, 1200 and 750. The distribution in the liver, spleen and blood was the same as in the small intestine. Remarkably, the maximum molecular mass of PA-DSS in each organ was below Mr 2000. Fig. 1E and F show the quantification of PA-DSS in each organ and its contents, respectively. The levels of PA-DSS were the highest in the liver 

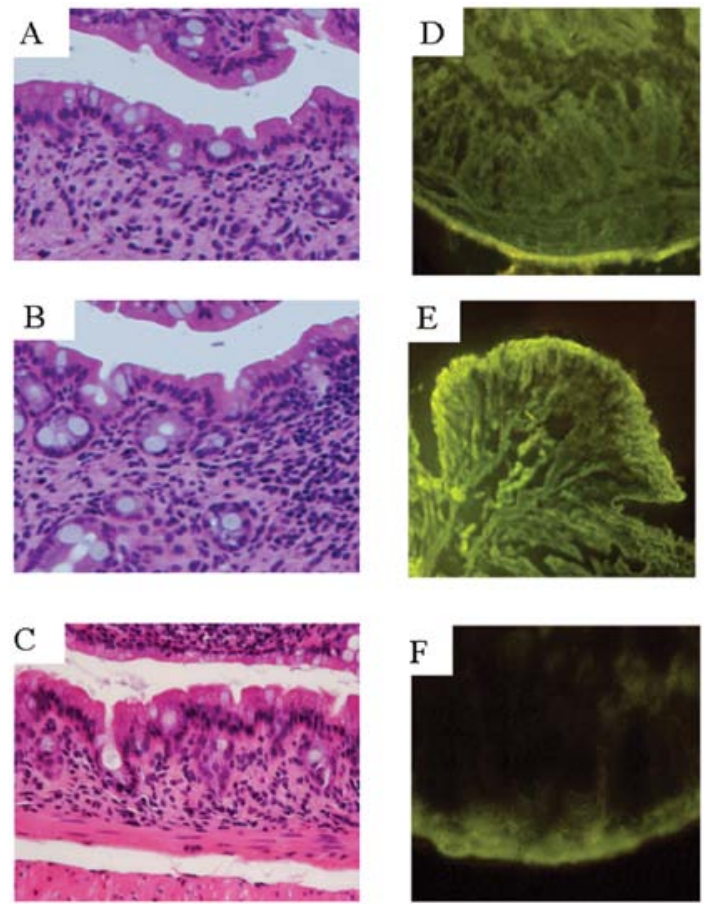

Figure 2. Mr 5000 PA-DSS-induced colitis. (A-C) Microscopic findings from the $\mathrm{H} \& \mathrm{E}$ staining or (D and $\mathrm{E}$ ) fluorescence microscopy of the colon in a $\mathrm{Mr}_{\mathrm{r}}$ 5000 PA-DSS-induced colitis mouse. (F) Fluorescence microscopy of the colon in a control mouse. (A-C) Original magnification, x100; (D-F), x40.

and spleen, and the concentration of PA-DSS in the contents of the small intestine reached $\sim 30 \%(\mathrm{w} / \mathrm{v})$. In the cecum, the concentration was $\sim 5 \%$ (w/w). Interestingly, this concentration was the same as in the diet.

Mr 5000 PA-DSS-induced colitis. Next, we observed DSS-induced colitis both macroscopically and microscopi- cally. After the Mr 5000 PA-DSS administration, diarrhea occurred on Days 3-4. Macroscopic examination of the colon revealed hyperemia, erosions and occasional tiny blood clots in the distal colon on Day 5. Using H\&E staining, there was obvious evidence of inflammatory cell infiltration into the mucosa and submucosa. Crypt shortening, entire crypt loss, and earthenware mortar-like deformity of the crypts, surface epithelial loss and mucosal edema were also evident (Fig. 2A-C). Fig. 2D and E show the fluorescence signals on Day 8. The signal increased significantly, and was localized throughout the entire intestinal wall as compared to the control mice, which had very weak signals localized mainly to the serosa (Fig. 2F). These results suggest that PA-DSS permeated the intestinal epithelium in a form under Mr 2000. In addition, PA-DSS subsequently invaded into the mucosa, submucosa, and muscle layers.

Mouse Mr 2500 PA-DSS-induced colitis. We next investigated whether this difference in the molecular mass distribution of PA-DSS in the lumen explain the fact that Mr 5000 PA-DSS mainly induces inflammation in the colon, but not in the stomach or small intestine. Specifically, we examined whether Mr 2500 PA-DSS could induce colitis or not. After the $\mathrm{Mr}_{\mathrm{r}}$ 2500 PA-DSS administration, the appearance of diarrhea was delayed until Days 6-7. The body weight loss was also less than that in mice fed Mr 5000 PA-DSS (Fig. 3A). This difference between Mr 2500 and 5000 DSS was statistically significant on Day 6. Mr 5000 PA-DSS-induced colitis was histologically more severe than Mr 2500 PA-DSS-induced colitis (Fig. 3B, control; C, Mr 2500 PA-DSS; and D, Mr 5000 PA-DSS). This difference between both groups was statistically significant (Fig. 3E). In this section, Mr 2500 PA-DSS was also depolymerized in the stomach. Interestingly, the majority of the PA-DSS in the cecal contents was also Mr 2500 DSS, at a concentration of $\sim 5 \%(\mathrm{w} / \mathrm{w})$, which was the same as that in the diet.
A

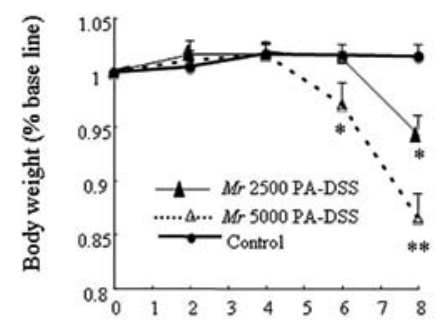

Duration of DSS administration (days)

\section{E}

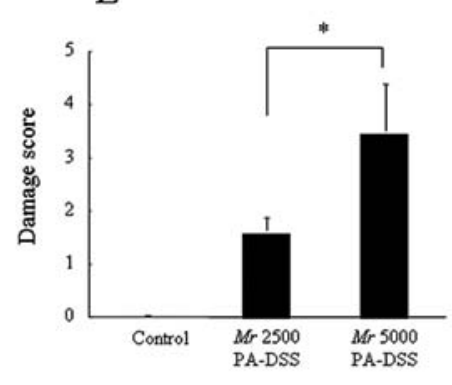

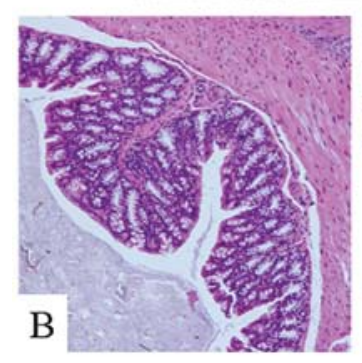
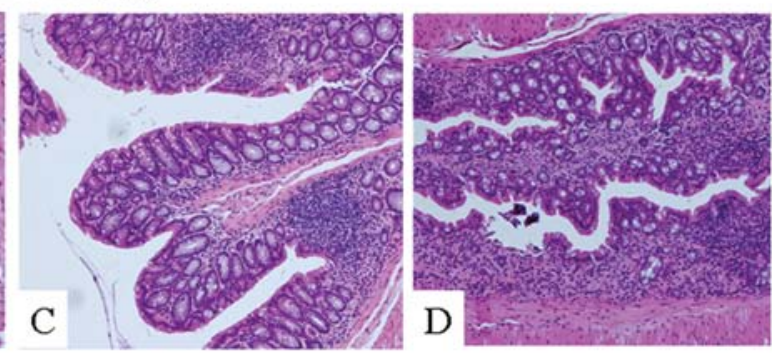

Figure 3. Mr 2500 PA-DSS-induced colitis. (A) Body weight losses in the control, $\mathrm{Mr}_{\mathrm{r}} 2500$ and 5000 DSS-induced colitis mice. (B-D) Microscopic findings of the colon in a control, $\mathrm{Mr}_{\mathrm{r}} 2500$ and 5000 DSS-induced colitis mice. Original magnification, x100 using H\&E staining. (E) The mucosal damage was quantified by a scoring system (12) in the control, $\mathrm{Mr}_{\mathrm{r}} 2500$ and $5000 \mathrm{DSS}$-induced colitis mice. All values are expressed as means $\pm \mathrm{SEM}$. ${ }^{*} \mathrm{P}<0.05$ vs. control and ${ }^{* *} \mathrm{P}<0.05$ vs. Mr 2500 or control. 

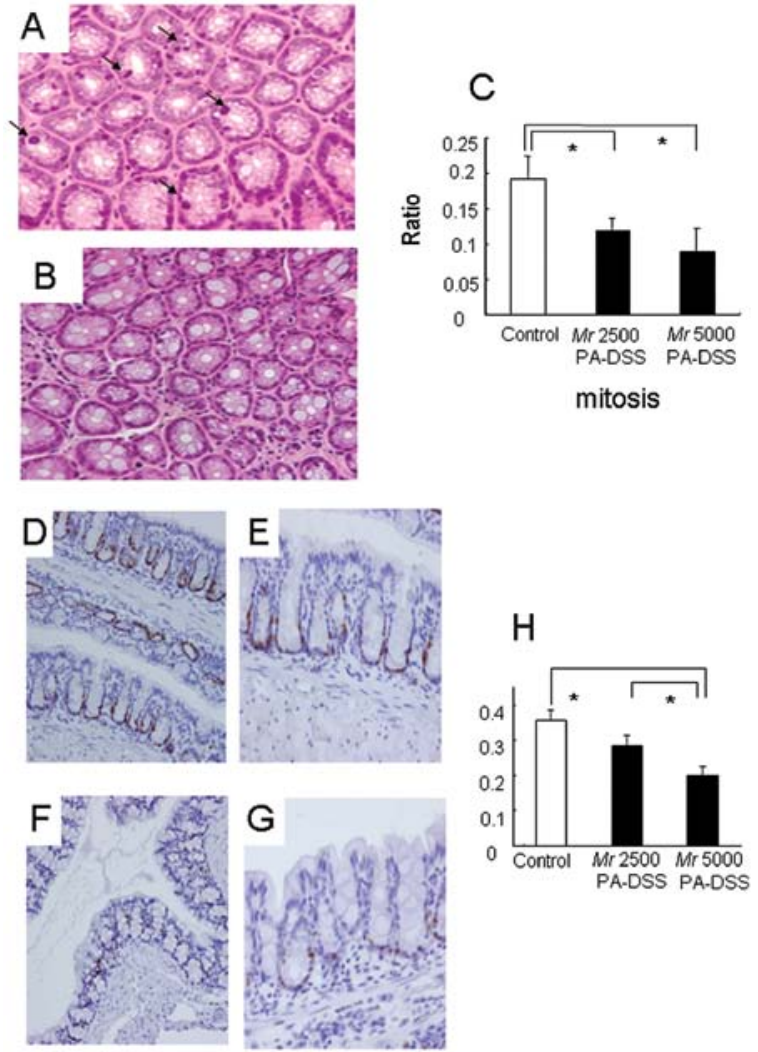

Figure 4. Mitosis and cell cycle in PA-DSS-induced colitis. (A) Microscopic findings of the colon in a control mouse. There was sporadic mitosis in the crypts (arrow). (B) Microscopic findings of the colon in a M 5000 DSSinduced colitis mouse. There were far fewer mitoses. (A and B) Original magnification, x200 using H\&E staining. (C) To quantify this mitotic activity, the ratio of the crypt counts with mitosis to the total cell count in the crypt was calculated. All values are expressed as means \pm SEM. ${ }^{*} \mathrm{P}<0.05$. (D and E) Immunohistochemical staining of the colon in a control mouse. There were many anti-Ki-67 immunopositive cells in the lower part of the crypt. ( $F$ and $\mathrm{G}$ ) Immunohistochemical staining in the colon of a $\mathrm{M}_{\mathrm{r}} 5000$ DSSinduced colitis mouse. There were far fewer anti-Ki-67 immunopositive cells in the lower part of the crypt. (D and F) Original magnification, x100 using anti-Ki-67 immunostaining. (E and G) Original magnification, $\mathrm{x} 200$ using anti-Ki-67 immunostaining. (H) To quantify these anti-Ki-67 immunopositive cells, the ratio of the crypt counts with the anti-Ki-67 immunopositive cell count to the total cell count in the crypt was calculated. All values are expressed as means \pm SEM. ${ }^{*} \mathrm{P}<0.05$.

Cell cycle arrest in PA-DSS-induced colitis. Subsequently, we examined how DSS contributes to the induction of colitis. We previously found that DSS strongly and rapidly inhibited the intracellular energy metabolism in Caco-2 cells (16). Therefore, we focused on the dynamics of the epithelial cells, especially the cell cycle status in this section.

In the control mice, there was sporadic mitosis present in the crypts using $\mathrm{H} \& \mathrm{E}$ staining (Fig. 4A). However, this mitosis was much less frequent in mice fed $\mathrm{Mr} 5000$ PA-DSS (Fig. 4B). To quantify this mitotic activity, the ratio of the cell count with mitosis to the total cell count in the crypt was calculated. Mr 5000 PA-DSS significantly reduced this mitosis ratio as compared to the control mice (Fig. 4C). With respect to $\mathrm{Mr} 2500 \mathrm{PA}-\mathrm{DSS}$, the reductions in the mitotic activity were somewhere between the control mice and the mice fed Mr 5000 PA-DSS.

On the other hand, we observed the cell cycle status of the colonic epithelium. Fig. 4D-G show the immunostaining using
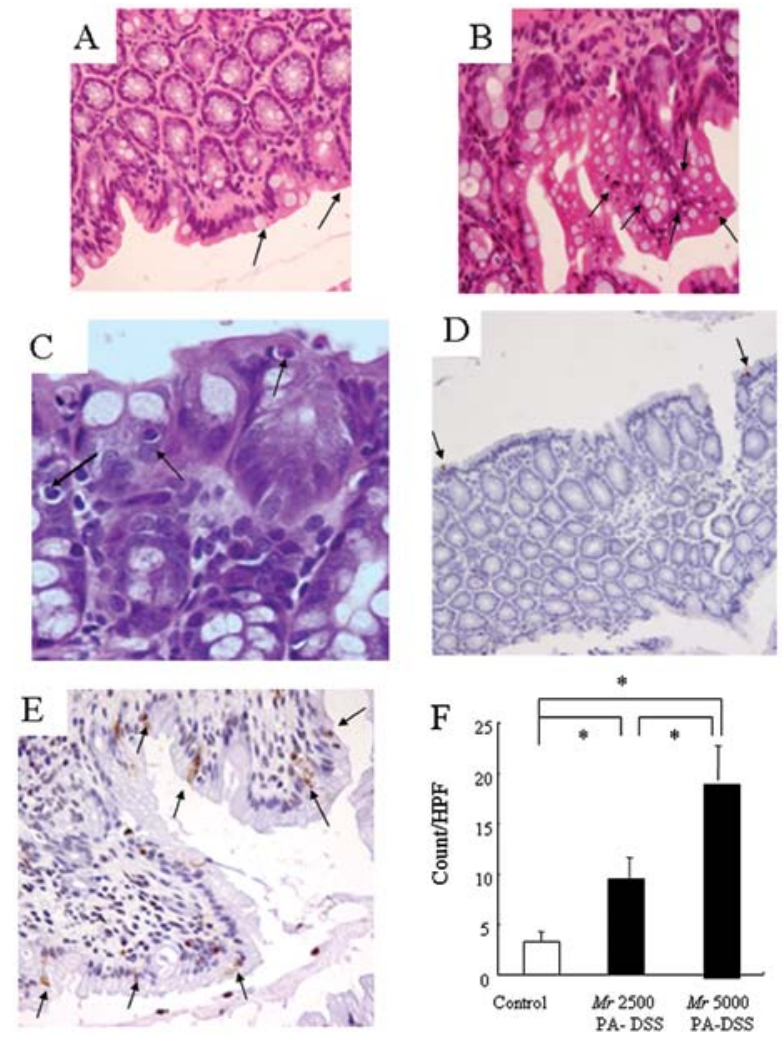

Figure 5. Apoptosis in the crypts in Mr 5000 or 2500 PA-DSS-induced colitis mice. (A) Microscopic findings of the colon in a control mouse. There were a few apoptotic bodies, especially at the top of the villi (arrow). (B and C) Microscopic findings of the colon in a $\mathrm{Mr}_{\mathrm{r}} 5000$ DSS-induced colitis mouse. There were far more apoptotic bodies recognized (arrow). (D) Immunohistochemical staining of the colon in a control mouse. There were a few TdT positive cells in the epithelium (arrow). (E) Immunohistochemical staining in the colon of a $\mathrm{Mr}_{\mathrm{r}} 5000 \mathrm{DSS}$-induced colitis mouse. There were far more TdT positive cells recognized (arrow). (A and B) Original magnification, $\mathrm{x} 100$, and (C) x400 using H\&E staining. (D and E) Original magnification, $\mathrm{x} 100$ using TdT staining. (F) To quantify these TdT positive cells, the ratio of the TdT positive cell count to the total cell count in the crypt was calculated. All values are expressed as means \pm SEM. ${ }^{*} \mathrm{P}<0.05$.

anti-mouse Ki-67 antibody, which labels all active phases of the cell cycle (G1, S, G2 and M phases). In the control mice, there were anti-Ki-67 immunopositive cells in the lower part of the crypt (Fig. 4D and E). However, these anti-Ki-67 immunopositive cells were far fewer in the lower part of the crypt in mice fed $\mathrm{Mr} 5000$ PA-DSS (Fig. 4F and G). To quantify these anti-Ki-67 immunopositive cells, the ratio of the antiKi-67 immunopositive cell count to the total cell count in the crypt was calculated. $\mathrm{Mr}_{\mathrm{r}} 5000$ PA-DSS significantly reduced the anti-Ki-67 immunopositive cell ratio as compared to the control mice (Fig. 4H). With respect to Mr 2500 PA-DSS, the reductions in the anti-Ki-67 immunopositive cell ratio was somewhere between the control mice and the mice fed Mr 5000 PA-DSS.

Apoptosis in PA-DSS-induced colitis. It has become clear that DSS arrested the cell cycle of the colonic epithelium. Therefore, we investigated whether DSS induces apoptosis of the colonic epithelium or not as the dynamics of the epithelial cells.

There were a few apoptotic bodies present, especially at the top of the villi, in the control mice (Fig. 5A). These apoptotic bodies were much more frequent in mice fed $\mathrm{Mr}_{\mathrm{r}} 5000$ 

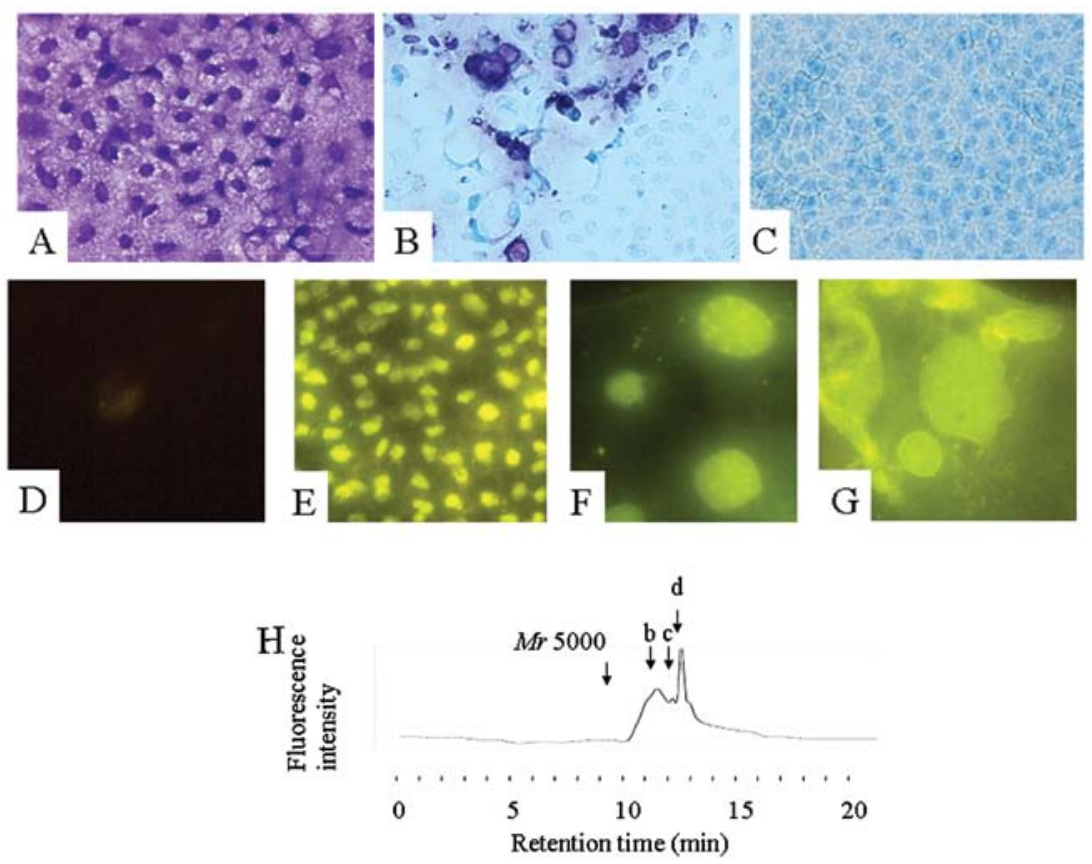

Figure 6. Metabolism of $\mathrm{Mr}_{\mathrm{r}} 5000$ PA-DSS in Caco-2 cells. (A) Toluidine blue staining of CaCo-2 cells incubated with 3\% Mr 5000 DSS for 24 h. (B) Toluidine blue staining of Caco-2 cells for $3 \mathrm{~h}$ after replacing with medium alone. (C) Toluidine blue staining of Caco- 2 cells for $24 \mathrm{~h}$ after replacing with medium alone. (D) Fluorescence microscopic image of control CaCo-2 cells. (E-G) Fluorescence microscopic images of CaCo-2 cells incubated with $3 \%$ Mr 5000 PA-DSS for 24 h. (A-C) Original magnification, x100; (D, F and G), x400; (E), x100. (H) Chromatogram of PA-DSS permeated outside a porous filter after 5 h. The molecular masses were approximately $\mathrm{Mr}_{\mathrm{r}} 1500$ (peak b), 1200 (peak c) and 750 (peak d), respectively. The data show the amount of Mr 5000 PA-DSS regardless of the degree of depolymerization.
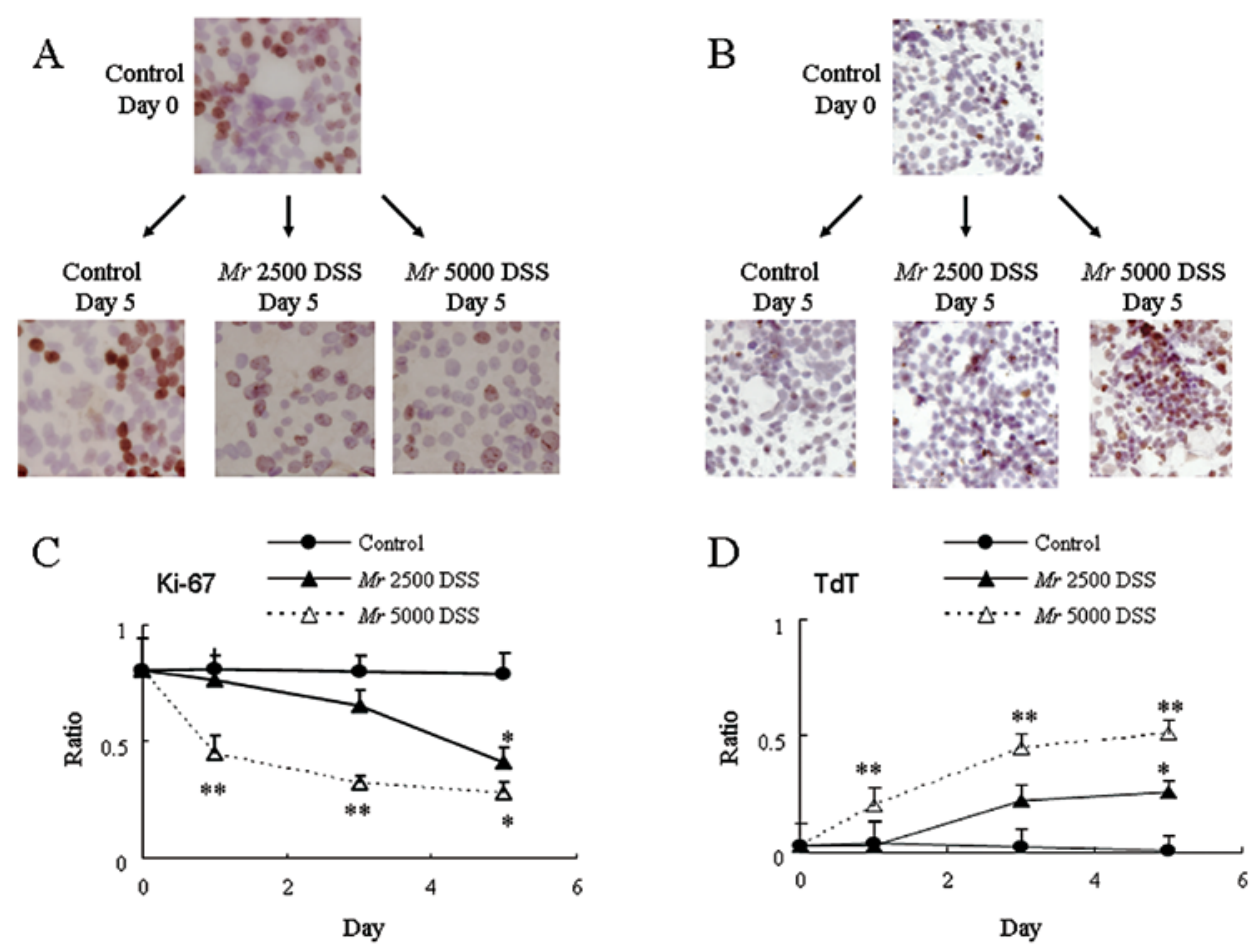

Figure 7. Cell cycle arrest and apoptosis in Caco-2 cells. Caco-2 cells were co-incubated with medium alone, 3\% Mr 5000 or 2500 DSS for 5 days. (A) Immunohistochemical staining of the Caco-2 cells using an anti-mouse Ki-67 antibody. (B) Immunohistochemical staining of Caco-2 cells using the TUNEL assay (TdT). (C and D) To quantify the anti-Ki-67 immunopositive cells or TdT positive cells, the ratio of the anti-Ki-67 immunopositive cell count or TdT positive cell count to the total cell count was calculated. All values are expressed as means \pm SEM. ${ }^{*} \mathrm{P}<0.05$ vs. control and ${ }^{* * *} \mathrm{P}<0.05$ vs. Mr 2500 or control.

PA-DSS (Fig. 5B and C). Fig. 5D and E show the staining using the TUNEL assay. In the control mice, there were a few TdT positive cells in the epithelium (Fig. 5D). However, these TdT-positive cells were far more frequent in mice fed Mr 5000 PA-DSS (Fig. 5E). We randomly calculated the TdT positive cell count in high power field (x400). $\mathrm{Mr} 5000$ 
PA-DSS significantly increased the number of TdT positive cells as compared to the control mice (Fig. 5F). With respect to $\mathrm{Mr}_{\mathrm{r}} 2500$ PA-DSS, the increase in the TdT positive cells was somewhere between the control mice and mice fed $\mathrm{Mr}_{\mathrm{r}} 5000$ PA-DSS. These results suggest that DSS essentially induced cell cycle arrest, especially at the $\mathrm{G}_{0}$ phase, and apoptosis of the colonic epithelial cells in vivo.

Metabolism of PA-DSS in Caco-2 cells. The molecular mass in the feces was predominantly $\mathrm{Mr} 5000$, whereas that in the mucosa of the colon was below $\mathrm{Mr}_{\mathrm{r}} 2000$. Does this result mean that some of the DSS was depolymerized when it passed through the epithelial cells? In addition, can DSS also induce cell cycle arrest and apoptosis in culture cells? We investigated the metabolism of PA-DSS using Caco-2 cells in order to clarify these possibilities. In general, a mixture of DSS and toluidine blue exhibits a color change from blue to violet called metachromasia. This color change is derived from the shift of the UV absorbance from 620 to $560 \mathrm{~nm}$ and a minimum molecular mass of 2,500 Da was required for a metachromatic reaction according to our previous study (13). Fig. 6A shows the toluidine blue staining of Caco-2 cells after $24 \mathrm{~h}$ of co-incubation with $\mathrm{Mr} 5000$ DSS in medium. The metachromatic reaction was present mainly in the nucleus. At $3 \mathrm{~h}$ after replacing with medium alone, the metachromatic reaction disappeared gradually (Fig. 6B) and was no longer detectable at $24 \mathrm{~h}$ (Fig. 6C). These findings strongly suggest that $\mathrm{Mr} 5000$ DSS entered the cells and was depolymerized to under $\mathrm{M}_{\mathrm{r}}$ 2500. Under a fluorescence microscope, the fluorescence intensity of the control CaCo-2 cells was very weak (Fig. 6D). $\mathrm{Mr}_{\mathrm{r}} 5000$ PA-DSS also entered the cells, and was bound mainly to the nucleus (Fig. 6E). On the surface of the nucleus, several more densely-bound areas were recognized (Fig. 6F). Some cells exhibited apoptotic features including nuclear shrinkage or fragmentation (Fig. 6G). The fluorescence intensity was also gradually reduced after replacing with medium alone.

The Mr 5000 PA-DSS was also depolymerized while it passed through the Caco-2 monolayer. Fig. $6 \mathrm{H}$ shows a chromatogram of PA-DSS in the medium outside of a porous filter at $5 \mathrm{~h}$ after $1 \% \mathrm{Mr} 5000$ PA-DSS in the medium was inserted inside the filter. At this time point, three peaks were recognized: approximately $\mathrm{Mr}_{\mathrm{r}} 1500$ (peak b), 1200 (peak c) and 750 (peak d), respectively. The $1 \% \mathrm{Mr} 5000$ PA-DSS solution did not reduce the TEER, indicating that $\mathrm{Mr}_{\mathrm{r}} 5000 \mathrm{PA}-\mathrm{DSS}$ passed through a transcellular and, not through paracellular pathway. These results confirmed that the $\mathrm{Mr}_{\mathrm{r}} 5000$ PA-DSS was depolymerized in Caco-2 cells.

Cell cycle arrest and apoptosis in Caco-2 cells. It has become clear that Caco-2 cells can also depolymerize PA-DSS. We thus investigated if DSS can also induce the cell cycle arrest and apoptosis in Caco-2 cells.

Fig. 7A shows the immunostaining using an anti-mouse Ki-67 antibody on Days 0 and 5 after co-incubation with $\mathrm{Mr} 5000$ or 2500 DSS in the medium. We calculated the ratio of the anti-Ki-67 immunopositive cell count to the total cell count in randomly-observed fields (Fig. 7C). These anti-Ki-67 immunopositive cells gradually decreased after co-incubation with 3\% Mr 5000 DSS in the medium. On the other hand, Fig. 7B shows the immunostaining using the TUNEL assay on Days 0 and 5 after co-incubation with $\mathrm{Mr} 5000$ or 2500 DSS in medium. We calculated the ratio of the TdT positive cell count to the total cell count in randomly-observed fields (Fig. 7D). These TdT positive cells gradually increased after co-incubation with 3\% Mr 5000 DSS in the medium. With respect to $3 \% \mathrm{Mr} 2500 \mathrm{DSS}$ in the medium, the reductions in the anti-Ki-67 immunopositive cell ratio and increase in the TdT positive cell ratio were somewhere between the control and co-incubation with 3\% $\mathrm{Mr} 5000$ DSS in the medium values. These results suggest that DSS also essentially induced cell cycle arrest, especially at the $\mathrm{G}_{0}$ phase, and apoptosis of Caco-2 cells in vitro.

\section{Discussion}

When investigating the pathogenesis of DSS-induced colitis, the metabolism of DSS is a problem that cannot be avoided. However, there has been no report that shows in detail the metabolites of DSS found in the biological materials, including the contents of the gut lumen. First, Mr 5000 PA-DSS was administer orally, and was rapidly depolymerized in the stomach. We previously reported that acidic but not alkaline conditions can depolymerize PA-DSS (13). In particular, $1 \mathrm{~N} \mathrm{HCl}$ was best suited for this reaction without depleting the sulfates in the molecule. Therefore, we speculated that the gastric acid might depolymerize the $\mathrm{Mr}_{\mathrm{r}} 5000$ PA-DSS. After the $\mathrm{Mr}_{\mathrm{r}} 5000$ PA-DSS was depolymerized in the gastric lumen, PA-DSS entered the lumen of the small intestine. The depolymerized PA-DSS concentration in the lumen of the small intestine reached $\sim 30$-fold greater than in the stomach contents. The amount of depolymerized PA-DSS in the wall of the small intestine was 3-fold greater than in the gastric mucosa. In addition, the molecular mass distribution of depolymerized PA-DSS in the blood, spleen, and liver was the same as the contents and mucosa of the small intestine. These results suggest that depolymerized PA-DSS could be easily absorbed in the small intestine. After entering the cecum, however, the molecular mass distribution was remarkably altered; contrary to our expectations, the Mr 5000 PA-SS became the main form. This $\mathrm{Mr}_{\mathrm{r}} 5000 \mathrm{PA}-\mathrm{SS}$ possibly escaped absorption in the small intestine. Regardless, it has become clear that $\mathrm{Mr}_{\mathrm{r}} 5000$ PA-DSS in the colon essentially strongly induced cell cycle arrest, especially at the $\mathrm{G}_{0}$ phase, and apoptosis of the colonic epithelial cells than depolymerized PA-DSS in vivo and in vitro. Therefore, this difference in the molecular mass distribution of PA-DSS in the lumen may explain the fact that PA-DSS mainly induces inflammation in the colon, but not in the stomach or the small intestine.

In addition, the intestinal epithelial cells are able to uptake and depolymerize PA-DSS. On the other hand, even Mr 500k DSS could enter Caco-2 cells, and the nucleus exhibited metachromasia (data not shown). DSS could also enter other types of cells, such as IEC-6 (a rat intestinal epithelial cell line) and Hep G2 (a human hepatoblastoma cell line) cells, and became depolymerized (data not shown). Since dextran (non-sulfated polysaccharide) cannot enter these cells nor the epithelium, these characteristics of DSS are quite remarkable. This DSS pathway, however, has not been clarified yet. In addition, it is still unclear how the DSS was depolymerized or what enzymes were involved. 
There have been a few previous reports showing in part the metabolism of DSS in the biological materials. In those reports, DSS was detected mainly using a metachromatic reaction (17-19). However, our method using the PA-labeling system seems more reliable. DSS depolymerized in the gut lumen cannot induce a metachromatic reaction any more because a minimum molecular mass of 2,500 Da is required for DSS to induce a metachromatic reaction according to our previous study (13).

In the present study, the precise mechanisms responsible for the induction of cell cycle arrest and apoptosis remain unclear. Some previous reports, however, have suggested an association between DSS and the cell cycle arrest. For example, the cell cycle of B lymphocytes was arrested by DSS (20). In this process, DSS inhibited the DNA topoisomerases, and controlled cell proliferation via DNA polymerase (21). This effect was the strongest at around $\mathrm{Mr} 4300$ (22). In addition, we previously reported that DSS strongly and rapidly inhibited reactive oxygen species (ROS) generation in Caco- 2 cells. In this study, ROS generation was reduced to $\sim 3 \%$ of the control value by $5 \mathrm{~h}$ after $1 \% \mathrm{DSS}$ exposure (16). The mechanisms responsible for this reduction in ROS generation remain unclear. This phenomenon, however, also suggests that DSS arrests intracellular energy metabolism, because ROS are generated during energy metabolism. Taken together, the early stage of DSS-induced colitis might involve a cytostatic mechanism (cellular metabolic arrest and cell cycle arrest), in other words, as if the cells were in hibernation.

With respect to apoptosis, a few previous reports showed the involvement of apoptosis in DSS-induced colitis (23). Since intestinal epithelial cells have a rapid cell cycle, it is logical to postulate that cell cycle arrest and apoptosis in addition to cellular metabolic arrest might lead to crypt shortening, entire crypt loss, and an earthenware mortar-like deformity of the crypt, and surface epithelial loss, which were partly reported by Cooper et al (24). Consequently, the mucosa might eventually develop breaks in the barrier of the epithelial cells as an early stage event.

It is also very interesting that $\mathrm{T}$ and $\mathrm{B}$ cells are not required for acute DSS-induced colitis, since DSS also produces colitis in severe combined immunodeficient (SCID) mice (25). Another previous study also suggests that DSS exhibits a direct toxicity against colonic epithelial cells, IEC-18 cells (26). Thereafter, the mucosal breaks would lead to mucosal inflammatory changes (24), for example through macrophage activation (27), the invasion of intestinal bacteria, or a variety of cytokine cascades. In that sense, many previous studies which reported that some growth hormones or factors ameliorated DSS-induced colitis (28-31) might be reasonable because these kinds of agents have been reported to promote the cell cycle $(32,33)$. On the other hand, Kitajima et al reported that the degree of colitis was stronger in the order of Mr 40k DSS $>$ Mr 5000 DSS in mice (17).

In addition, carrageenan is produced from seaweed and is used as a gelling agent in food. Carrageenan is another type of sulfated polysaccharide consisting of anhydro-D-galactose ( $\alpha$-1,3-galactosidic link) and sulfate (over Mr 100k to 800k), and also induces colitis (34). Carrageenan also arrested the cell cycle (35). Interestingly, the acid-degraded form of carra- geenan (poligeenan, $\mathrm{Mr}$ 40k) more strongly caused colitis. It is also said that a critical DSS load exceeding $30 \mathrm{mg} / \mathrm{g}$ body wt was required to induce colitis (36). Taken together, the most important finding might be that a certain amount of DSS with a certain range of molecular masses is required to induce cell cycle arrest and apoptosis after the DSS is depolymerized in the gut lumen, and subsequently enters the cells. These results might attract the interest of many researchers. When focusing on the etiology of IBS or other types of colitis, including DSS-induced colitis, other kinds of materials may induce the cytostatic effects, apoptosis and eventually colitis.

Finally, several types of bacteria, including Pseudomonas carrageenovora of marine origin, have been identified which are able to hydrolyze carrageenan (37). We also reported that DSS was depolymerized by Proteus species in the gut lumen (38). It is possible that some kinds of bacteria in the gut lumen may depolymerize the DSS and modulate the DSS-induced colitis.

In conclusion, we proposed one plausible mechanism responsible for an early stage event in DSS-induced colitis. DSS is depolymerized rapidly in the mouse stomach. This depolymerized DSS cannot induce severe inflammation in the stomach or small intestine. However, the majority of the DSS in the colonic lumen returned to the Mr 5000 form. This Mr 5000 DSS induced even more severe colitis through cell cycle arrest and apoptosis than the depolymerized DSS.

\section{References}

1. Fiocchi C: Inflammatory bowel disease: etiology and pathogenesis. Gastroenterology 115: 182-205, 1998.

2. Bernstein CN, Blanchard JF, Kliewer E and Wajda A: Cancer risk in patients with inflammatory bowel disease: a populationbased study. Cancer 91: 854-862, 2001.

3. Elson CO, SartorRB, Tennyson GS and Riddell RH: Experimental models of inflammatory bowel disease. Gastroenterology 109: 1344-1367, 1995

4. Watt $J$ and Marcus R: Ulceration of the colon in rabbits fed sulfated amylopectin. J Pharm Pharmacol 24: 68-69, 1972.

5. Ricketts CR: Dextran sulphate-a synthetic analogue of heparin. Biochem J 51: 129-133, 1952.

6. Tanaka T, Kohno H, Suzuki R, Yamada Y, Sugie S and Mori H: A novel inflammation-related mouse colon carcinogenesis model induced by azoxymethane and dextran sodium sulfate. Cancer Sci 94: 965-973, 2003.

7. Suzuki R, Kohno H, Sugie S and Tanaka T: Sequential observations on the occurrence of preneoplastic and neoplastic lesions in mouse colon treated with azoxymethane and dextran sodium sulfate. Cancer Sci 95: 721-727, 2004.

8. Yamada M, Ohkusa T and Okayasu I: Occurrence of dysplasia and adenocarcinoma after experimental chronic ulcerative colitis in hamsters induced by dextran sulphate sodium. Gut 33: 1521-1527, 1992.

9. Okayasu I, Hatakeyama S, Yamada M, Ohkusa T, Inagaki Y and Nakaya R: A novel method in the induction of reliable experimental acute and chronic ulcerative colitis in mice. Gastroenterology 98: 694-702, 1990.

10. Araki Y, Andoh A, Fujiyama Y, Hata K, Makino J, Okuno T, Nakanura F and Bamba T: Application of 2-aminopyridine fluorescence labeling in the analysis of in vivo and in vitro metabolism of dextran sulfate sodium by size-exclusion highperformance liquid chromatography. J Chromatogr B Biomed Sci Appl 753: 209-215, 2001.

11. Arak Y, Andoh A, Fujiyama Y, Hata K, Makino J, Shimada M, Bamba H, Okuno T, Urushiyama $\mathrm{N}$ and Bamba T: Analysis of $\alpha$-amylase-derived pyridylamino-dextran sulfate oligomers by the combination of size-exclusion and reversed-phase highperformance liquid chromatography. J Chromatogr B Analyt Technol Biomed Life Sci 766: 351-356, 2002. 
12. Araki Y, Kanauchi O, Sugihara H, Fujiyama Y and Hattori T: Germinated barley foodstuff suppresses dextran sulfate experimental colitis in rats: the role of mast cells. Int J Mol Med 19: 257-262, 2007.

13. Araki Y, Mukaisyo K, Sugihara H and Hattori T: Decomposition of dextran sulfate sodium under alkaline, acidic, high temperature and high pressure conditions. Oncol Rep 20: 147-149, 2008.

14. Hidalgo IJ, Raub TJ and Borchardt RT: Characterization of human colon carcinoma cell line (Caco-2) as a model system of intestinal epithelial permeability. Gastroenterology 96: 736-749, 1989.

15. Araki Y, Katoh T, Ogawa A, Bamba S, Andoh A, Koyama S, Fujiyama Y and Bamba T: Bile acid modulates transepithelial permeability via the generation of reactive oxygen species in the Caco-2 cell line. Free Radic Biol Med 39: 769-780, 2005.

16. Araki Y, Sugihara H and Hattori T: In vitro effects of dextran sulfate sodium on a Caco-2 cell line and plausible mechanisms for dextran sulfate sodium-induced colitis. Oncol Rep 16: 1357-1362, 2006.

17. Kitajima S, Takuma $S$ and Morimoto M: Histological analysis of murine colitis induced by dextran sulfate sodium of different molecular weights. Exp Anim 49: 9-15, 2000.

18. Hoshi O, Iwanaga T and Fujino MA: Selective uptake of intraluminal dextran sulfate sodium and senna by macrophages in the cecal mucosa of the guinea pig. J Gastroenterol 31: 189-198, 1996.

19. Hiebert LM, Wice SM, Jaques LB, Williams KE and Conly JM: Orally administered dextran sulfate is absorbed in HIV-positive individuals. J Lab Clin Med 133: 161-170, 1999.

20. Burg DL, Harrison ML and Geahlen RL: Cell cycle-specific activation of the PTK72 protein-tyrosine kinase in B lymphocytes. J Biol Chem 68: 2304-2306, 1993.

21. Umemura K, Yanase K, Suzuki M, Okutani K, Yamori T and Andoh T: Inhibition of DNA topoisomerases I and II, and growth inhibition of human cancer cell lines by a marine microalgal polysaccharide. Biochem Pharmacol 66: 481-487, 2003.

22. Furukawa $K$ and Bhavanandan VP: Influences of anionic polysaccharides on DNA synthesis in isolated nuclei and by DNA polymerase alpha: correlation of observed effects with properties of the polysaccharides. Biochim Biophys Acta 740: 466-475, 1983.

23. Vetuschi A, Latella G, Sferra R, Caprilli R and Gaudio E: Increased proliferation and apoptosis of colonic epithelial cells in dextran sulfate sodium-induced colitis in rats. Dig Dis Sci 47: 1447-1457, 2002.

24. Cooper HS, Murthy SN, Shah RS and Sedergran DJ: Clinicopathologic study of dextran sulfate sodium experimental murine colitis. Lab Invest 69: 238-249, 1993.

25. Dieleman LA, Ridwan BU, Tennyson GS, Beagley KW, Bucy RP and Elson CO: Dextran sulfate sodium-induced colitis occurs in severe combined immunodeficient mice. Gastroenterology 107: $1643-1652,1994$.
26. Ni J, Chen SF and Hollander D: Effects of dextran sulfate sodium on intestinal epithelial cells and intestinal lymphocytes. Gut 39: 234-241, 1996.

27. Yoshino T, Nakase H, Honzawa Y, Matsumura K, Yamamoto S, Takeda Y, Ueno S, Uza N, Masuda S, Inui K and Chiba T: Immunosuppressive effects of tacrolimus on macrophages ameliorate experimental colitis. Inflamm Bowel Dis 16: 2022-2033, 2010.

28. Zhao J, de Vera J, Narushima S, Beck EX, Palencia S, Shinkawa P, Kim KA, Liu Y, Levy MD, Berg DJ, Abo A and Funk WD: R-spondin1, a novel intestinotrophic mitogen, ameliorates experimental colitis in mice. Gastroenterology 132: 1331-1343, 2007.

29. Egger B, Carey HV, Procaccino F, Chai NN, Sandgren EP, Lakshmanan J, Buslon VS, French SW, Büchler MW and Eysselein VE: Reduced susceptibility of mice overexpressing transforming growth factor alpha to dextran sodium sulfate induced colitis. Gut 43: 64-70, 1998.

30. Williams KL, Fuller CR, Dieleman LA, DaCosta CM, Haldeman KM and Sartor RB and Lund PK: Enhanced survival and mucosal repair after dextran sodium sulfate-induced colitis in transgenic mice that overexpress growth hormone. Gastroenterology 120: 925-937, 2001.

31. Tessner TG, Cohn SM, Schloemann S and Stenson WF: Prostaglandins prevent decreased epithelial cell proliferation associated with dextran sodium sulfate injury in mice. Gastroenterology 115: 874-882, 1998.

32. Lepique AP, Moraes MS, Rocha KM, Eichler CB, Hajj GN Schwindt TT and Armelin HA: c-Myc protein is stabilized by fibroblast growth factor 2 and destabilized by ACTH to control cell cycle in mouse Y1 adrenocortical cells. J Mol Endocrinol 33: 623-638, 2004.

33. Slominski A, Zbytek B, Pisarchik A, Slominski RM, Zmijewski MA and Wortsman J: CRH functions as a growth factor/cytokine in the skin. J Cell Physiol 206: 780-791, 2006.

34. Watt $\mathbf{J}$ and Marcus R: Carrageenan induced ulceration of the large intestine in the guinea pig. Gut 12: 164-171, 1991.

35. Ling KY, Bhalla D and Hollander D: Mechanisms of carrageenan injury of IEC18 small intestinal epithelial cell monolayers. Gastroenterology 95: 1487-1495, 1988.

36. Vowinkel T, Kalogeris TJ, Mori M, Krieglstein CF and Granger DN: Impact of dextran sulfate sodium load on the severity of inflammation in experimental colitis. Dig Dis Sci 49: 556-564, 2004.

37. McLean MW and Williamson FB: kappa-Carrageenase from Pseudomonas carrageenovora. Eur J Biochem 93: 553-558, 1979.

38. Araki Y, Mukaisho K, Sugihara H, Fujiyama Y and Hattori T: Proteus mirabilis sp. intestinal microflora grow in a dextran sulfate sodium-rich environment. Int J Mol Med 25: 203-208, 2010. 